\title{
Aspects Bacteriologiques Des Infections Du Site Operatoire Au Centre Hospitalier Departemental Du Borgou A Parakou (Benin)
}

\section{Hodonou Montcho Adrien}

Faculté de Médecine de l'Université de Parakou ; Service de chirurgie générale, Centre Hospitalier Départemental du Borgou, Parakou, Bénin

\section{Hounkponou Fany}

Faculté de Médecine de l'Université de Parakou ; Service de gynécologieobstétrique, Centre Hospitalier Départemental du Borgou, Parakou, Bénin

\section{Allodé Salako Alexandre Tobome Sêmêvo Romaric Fatigba Olatoundji Holden \\ Tamou Sambo Bio Mensah Emile Antoine Dodji}

Faculté de Médecine de l'Université de Parakou ; Service de chirurgie générale, Centre Hospitalier Départemental du Borgou, Parakou, Bénin

\section{Atakpa Félix}

Service de pharmacie, Centre Hospitalier Départemental Borgou

\section{Akpata Raphael}

\section{Bankolé Emilie}

Service de médecine interne,

Hôpital de Zone Saint Jean de Dieu de Tanguiéta, Bénin

\section{Dadjo Yves}

Service de Chirurgie traumatologique et orthopédique,

Hôpital d'Instruction des Armées de Parakou, Bénin

\section{Mêhinto Kouassi Delphin.}

Faculté des Sciences de la Santé de l'Université d'Abomey-Calavi ; Service de chirurgie viscérale A, Centre National Hospitalier et Universitaire Hubert Koutoukou Maga, Cotonou, Bénin

\section{Abstract}

Objective : Describe the bacteriological aspects of surgical site infections (SSI) at the regional hospital centre Borgou. 
Methods : From a descriptive, prospective study from february 2013 to july 2014, about 603 patients operated during the recrutment period(six mounths), the bacteriological data of 44 cases of SSI diagnosed according to the criteria CDC/NHSN have been analysed. Results : The frequency of SSI was $7.3 \%$ of the operated (44/603). The superficial incisional infections were 6 , the deep infections were 34 and the organ infections or infections of space were 4 . The sampling culture of the liquid was negative with 12 cases (27.3\%) and positive with 32 cases (72.7\%). Two germs were isolated in two cases. The negative Gram germs were the most isolated with 22 cases (64.7\%) of Escherichia coli. The multiresistance was noted with 14 cases out of $34(41.2 \%)$.

Conclusion : The negative Gram germs were those which predominate in the infections of the operating site at the regional hospital center Borgou. The high rate of resistance to usual antibiotics must raise preventive and hard actions in order to inflect the graph of frequency and protect the antibiotics.

Keywords : Infection, surgical site, Antibiogram, negative Gram

\section{Resume}

Objectif : décrire les aspects bactériologiques des infections du site opératoire au centre hospitalier départemental du Borgou

Méthodes : à partir d'une étude prospective, descriptive de février 2013 à juillet 2014,sur 603 malades opérés dans la période de recrutement, les données bactériologiques de 44 cas d'ISO diagnostiqués selon les critères des CDC/NHSN ont été analysées.

Résultats : la fréquence des infections du site opératoire était de $7,3 \%$ des opérés (44/603). Les infections incisionnelles superficielles étaient 6, les profondes 34 et les infections d'organe ou d'espace étaient 4 . La culture du prélèvement du liquide était stérile dans 12 cas $(27,3 \%)$ et positive dans 32 cas $(72,7 \%)$. Dans deux cas étaient isolés deux germes. Les germes Gram négatifs étaient les plus isolés avec 22 cas $(64,7 \%)$ de Escherichia coli. La multirésistance était notée dans 14 cas sur 34 (41,2\%).

Conclusion : les germes Gram négatifs étaient les germes qui prédominent dans les infections du site opératoire au centre hospitalier départemental du Borgou. Le fort taux de résistance aux antibiotiques usuels doit susciter des actions préventives ardies pour infléchir la courbe de fréquence et protéger les antibiotiques.

Mots clés : Infection, Site opératoire, Antibiogramme, Gram négatif 


\section{Introduction}

L'infection du site opératoire (ISO) est une infection incisionnelle, d'organe ou d'espace, survenant dans les 30 jours suivant l'intervention ou dans l'année en cas de mise en place d'un implant ou d'un matériel prothétique [ Horan et al.,1992, Francoli et al.,1996]. Malgré les progrès de la technique chirurgicale, de l'antibiothérapie et des mesures thérapeutiques récentes, l'infection est encore un problème majeur en chirurgie [Ruef et $a l ., 1998]$. Dans les pays en voie de développement, l'ISO est l'une des infections associées aux soins les plus fréquentes [Aiken et al.,2013] et constitue une hantise pour le staff chirurgical. Les voies de transmission des micro-organismes ne sont pas parfaitement connues ; toutefois, les microorganismes proviennent pour la plupart de la flore microbienne du patient lui-même (source endogène à $90 \%$ ) et plus précisément du site incisionnel ( $95 \%$ de la source endogène) [Francoli et al., 1996]. Le germe responsable de l'ISO varie d'un environnement à un autre, d'un service à un autre et d'un type de chirurgie à un autre. Identifier les bactéries responsables des ISO dans les services chirurgicaux du Centre Hospitalier Départemental du Borgou (CHD-Borgou) et étudier leur sensibilité a été l'objectif de la présente étude afin de permettre l'orientation des antibioprophylaxies et au besoin de la stratégie des antibiothérapies probabilistes.

\section{Cadre et methodes}

Il s'agissait d'une étude descriptive avec recueil prospectif des données, effectuée dans les services de chirurgie générale et de gynécologieobstétrique du Centre Hospitalier Départemental du Borgou du $1^{\text {er }}$ février2013 au 31 juillet 2014. Les deux services sont les seuls à avoir de blocs opératoires. Les activités opératoires de gynécologie et d'obstétrique se déroulent dans le bloc de la maternité, situé à trois cents mètres environ du bloc de chirurgie qui reçoit les cas opératoires de toutes les autres spécialités chirurgicales. Les mêmes anesthésistes officient dans les deux blocs. Le linge et le matériel sont traités et stérilisés dans les mêmes conditions. Le recrutement des cas d'ISO était exhaustif et avait pris en compte tous les malades opérés dans les deux services sur une durée 6 mois (de février à juillet 2013). La surveillance des malades ainsi recrutés était poursuivie sur un mois après l'intervention ou sur un an selon qu'il a été fait ou non usage d'implant. Etaient inclus, tous les patients ayant été opérés dans l'un des deux blocs opératoires durant la période de recrutement, ayant donné leur consentement et qui avaient présenté une ISO dans les trente jours ou un an post-opératoires. Les critères diagnostiques étaient ceux de "Centers for Disease Control and Prevention/National Healthcare Safety Network (CDC/NHSN) » de mars 2009 [Horan et al.,2008]. Dès qu'une plaie opératoire répondait auxdits critères, on procédait au prélèvement par 
écouvillonnage. Le laboratoire de microbiologie du CHD, laboratoire de référence dans les départements du Borgou et de l'Alibori a servi de cadre pour les manipulations biologiques. Au laboratoire étaient réalisés un examen direct du prélèvement à l'état frais au microscope optique puis un autre après la coloration au Gram. Le liquide d'infection est ensuite ensemencé sur les différents milieux de culture à savoir le milieu Chapman Agar Salt, le milieu Eosin Methylene Blue Agar (EMB), le milieu à la gélose au sang de mouton et le milieu à la gélose chocolat. Ces milieux ensemencés sont mis à l'étuve pendant 24 heures. Quand un germe était identifié, une de ses colonies était repiquée sur du milieu Mueller Hinton en vue de l'antibiogramme. La lecture se faisait 24 heures après. Les données ainsi obtenues ont été analysées grâce au logiciel Epi info 7.1.1.14.

\section{Resultats}

Du $1^{\text {er }}$ février au 31 juillet 2013, 603 patients ont été opérés au CHDB (218 patients en chirurgie générale dont 155 hommes pour 63 femmes et 385 en gynécologie obstétrique). L'âge moyen était de $27,1 \pm 13,0$ ans. Quarante-quatre (28 de chirurgie et 16 de gynécologie-obstétrique) ont présenté d'ISO et tous ont accepté participer à notre étude. Notre étude a alors porté sur ces 44 patients et chacun n'avait qu'une seule ISO. La fréquence de l'ISO au CHD-B a été donc de 7,3\% (44/603). L'âge moyen des patients atteints d'ISO était de 30,7 $\pm 15,8$ ans avec des extrêmes de 5 mois et 70 ans. En chirurgie générale, les 28 patients atteints d'ISO se répartissaient en 23 hommes et 5 femmes.

Les pathologies dont les suites étaient compliquées d'ISO chez les 44 patients étaient :

- $\quad$ chirurgie viscérale (14 cas ; 31,8\%) : péritonite aigüe généralisée (5 cas), abcès appendiculaire ( 4 cas), hernie ( 2 cas), occlusion intestinale (1 cas), lipome sous cutané ( 1 cas), appendicite aigüe ( 1 cas) ;

obstétrique (14 cas ; 31,8\%): souffrance fœtale aigüe (5 cas), dystocie (4 cas), syndrome de pré rupture ( $1 \mathrm{cas})$, infection ovulaire (1 cas), hémoglobinopathie sur grossesse (1 cas), mort fœetale in utéro (1 cas), utérus bi-cicatriciel (1 cas) ;

- $\quad$ chirurgie orthopédique (8 cas ; 18,2\%) : fracture de membre (4 cas), écrasement de membre ( 3 cas), ostéomyélite chronique ( 1 cas) ; - neurochirurgie ( 5 cas ; 11,4\%) : canal lombaire rétréci ( 1 cas), hernie discale ( 1 cas), fracture de vertèbre ( 1 cas), myéloméningocèle (1cas), hydrocéphalie tétraventriculaire (1 cas) ;

- gynécologie (2 cas; 4,5\%) : tumeur maligne du sein et tumeur maligne de l'endomètre ;

chirurgie urologique (1 cas ; 2,3\%) : adénome de la prostate. 
Il y avait 34 cas d'ISO incisionnelle profonde; 6 cas d'ISO incisionnelle superficielle et 4 cas d'ISO d'organe ou d'espace.

Le résultat des examens bactériologiques des prélèvements étaient négatifs chez 12 patients (27,3\%) et positifs chez 32 (72,7\%). Quatre types de germes ont été retrouvés à savoir: Escherichia coli 22 (64,7\%); Pseudomonas aeruginosa 7 (20,6\%); Staphylococcus epidermidis 4 (11,8\%) ; Proteus mirabilis 1 (2,9\%). Chez deux patients, on avait retrouvé deux germes différents ce qui porte le nombre total d'isolats à 34 .

La répartition des germes en fonction des types d'ISO se présentait comme suit :

- $\quad$ ISO incisionnelle superficielle : Escherichia coli (2/4); Pseudomonas aeruginosa (1/4) ; Staphylococcus epidermidis (1/4).

- $\quad$ ISO incisionnelle profonde : Escherichia coli (16/32); Pseudomonas aeruginosa (6/32); Staphylococcus epidermidis (3/32) ; Proteus mirabilis (1/32).

- $\quad$ ISO d'organe/espace : Escherichia coli (4/4).

Dans $41,2 \%$ des cas de culture positive (14/34), aucune sensibilité in vitro aux antibiotiques utilisés n'a été notée. Il s'agissait de : 10 cultures pour Escherichia coli; 2 cultures pour Pseudomonas aeruginosa ; une culture pour Staphylococcus epidermidis et une culture pour Proteus mirabilis.

Les activités des antibiotiques utilisés au laboratoire sur les différents types de germes se présentaient comme l'indique le tableau I.

Tableau : Activités des antibiotiques utilisés au laboratoire sur les germes identifiés.

\begin{tabular}{|c|c|c|}
\hline Agent pathogène & Antibiotiques sensibles & Antibiotiques résistants \\
\hline Escherichia coli & Péfloxacine ; Nétilmycine & $\begin{array}{l}\text { Ciprofloxacine ; Amoxi-clav } \\
\text { Ceftriaxone ; Gentamycine }\end{array}$ \\
\hline $\begin{array}{l}\text { Pseudomonas } \\
\text { aeruginosae }\end{array}$ & Péfloxacine ; Norfloxacine & $\begin{array}{c}\text { Ciprofloxacine ; Amoxi-clav } \\
\text { Ceftriazone }\end{array}$ \\
\hline $\begin{array}{l}\text { Staphyloccocus } \\
\text { epidermidis }\end{array}$ & Amoxi-clav ; Nétilmycine & $\begin{array}{l}\text { Oxacilline ; Gentamycine ; } \\
\text { Ampicilline }\end{array}$ \\
\hline Proteus mirabilis & Aucun & $\begin{array}{l}\text { Ofloxacine ; Péfloxacine } \\
\text { Ceftriaxone ; Nétilmycine }\end{array}$ \\
\hline
\end{tabular}

\section{Discussion}

L'infection du site opératoire est une réalité au CHD-B avec une fréquence de 7,3\%. Ce même taux a été rapporté par Giri et al. [Giri et al.,2008] mais il est supérieur aux 2.0\% retrouvés par Oni et al. [Oni et al.,2006]. D'autres auteurs ont rapportés des fréquences plus élevées [Makoutodé et al.,2002 ; Hernandez et al.,2005; Umesh et al.,2008]. La fréquence de l'ISO est le reflet de la qualité des soins chirurgicaux [Walter et al. ;2008 ;Satyanarayana et al.,2011 ], eux-mêmes tributaires de plusieurs facteurs dont, la qualité des infrastructures et équipements, la formation et la conscience professionnelle des ressources humaines et aussi du comportement des demandeurs des soins. 
Le facteur étiologique de l'ISO étant le germe, tous les patients atteints d'ISO ont bénéficié de prélèvement de liquide d'infection à partir duquel l'examen bactériologique a été réalisé et nous avons observé que dans 27,3\%, le liquide était stérile. Alberto et al. [Alberto et al.,2009], Chadli et al. [Chadli et al.,2005] ont rapporté aussi des cas de culture stérile. L'antibiothérapie systématique a été de règle chez ces opérés étant donné les contextes de travail (malades souvent reçus en urgence pour chirurgie sale infectée ou contaminée) .

Les germes retrouvés étaient pour la plupart des gram-négatifs dont le plus fréquent était Escherichia coli $(64,7 \%)$. En effet, pour 11 patients $(25,0 \%)$ des patients atteints d'ISO, la pathologie pourvoyeuse intéressait les anses intestinales. Aussi, selon le rapport du comité d'expert du National Nosocomial Infections Surveillance (NNIS) des USA [NNIS system, 1996], les germes gram-négatifs et d'autres micro-organismes entériques pouvaientils coloniser la peau à certains sites, y compris l'aisselle, le périnée et l'aine. Les patients présentant des incisions à ces sites pouvaient avoir une ISO causée par des germes gram-négatifs. Par ailleurs, nous avons remarqué que tous les cas d'ISO d'organe ou d'espace étaient causés par Escherichia coli (4/4). Les germes gram-négatifs sont identifiés première cause d'ISO dans d'autres études [Ouedraogo et al.,2011;Barbut et al.,2004; Al-Hajje et al.,2012]. En revanche, certains auteurs ont rapporté la prédominance des germes gram-positifs [Alberto et al.,2009; Wassef et al.,2012]. Le taux de multirésistance des bactéries retrouvés était de $41,2 \%$ pour l'ensemble des prélèvements comportant de germes. Ce taux était supérieur à celui de $25,4 \%$ rapporté par Wassef et al. [Wassef et al.,2012] mais il était inférieur au 63.9\% rapporté par Hernandez et al. [Hernandez et al.,2005]. Cette multirésistance élevée confirme qu'il s'agit bien de germes hospitaliers. Elle doit faire changer complètement la politique non seulement d'antibioprophylaxie dans les services chirurgicaux de l'hôpital mais également des protocoles d'antibiothérapie probabiliste.

\section{Conclusion:}

L'infection du site opératoire au CHD-B est souvent causée par des germes gram-négatifs en particulier l'Escherichia coli qui développe de plus en plus de résistance aux antibiotiques couramment utilisés dans le service. Pour préserver ce capital précieux que constituent les antibiotiques, il urge de développer des stratégies rigoureuses à travers des protocoles d'antibioprophylaxie et d'antibiothérapie probabiliste. Une vigilance doit être observée par la répétition périodique de cette étude. 


\section{References:}

Aiken AM, Wanyoro AK, Mwangi J, Mulingwa P, Mulingwa P, Wanjohi J, et al. 2013 Evaluation of surveillance for surgical site infections in Thika Hospital, Kenya. J Hosp Infect; 83(2):140-5.

Alberto DL, Silvano P, Francesco R, Alberto M, Elena P, Vincenzo P, et al. 2009 Surgical site infection in an Italian surgical ward: A prospective study. Surgical Infections; 10(16):533-8.

Al-Hajje A,Ezedine M, Hammoud H, Awada S, Rachidi S,Zein S, Salameh P. 2012 Aspects actuels des infections nosocomiales au Centre Hospitalier Libanais de Beyrouth. Revue de Santé de la Méditeranée Orientale ; 18(5):495-500

Barbut F,CarbonneB, Truchot F, Spielvogel C, Jannet D, Goderel I, Lejeune V, Milliez J. 2004 Infections du site opératoire chez les patientes césarisées : bilan de cinq années de surveillance. J Gynecol Obstet Biol Reprod; $33: 487-96$

Chadli M, Rtabi N, Alkandry S, Kock JL Achour A, Buisson Y, et al. 2005 Incidence des infections du site opératoire : étude prospective à l'hôpital militaire d'instruction de Mohamed V de Rabbat, Maroc. Med Mal Infect;35(4):218-22.

Francoli P, Nahimana I, Widmer A. 1996 Infection du site chirurgical. SwissNoso;3(1):1-15

Giri BR, Pant HP, Sreeramareddy CT, Sen PK. 2008 Surgical site infection and antibiotics use pattern in a tertiary care hospital in Nepal. P Pak Med Asso;58(3):148-51.

Hernandez K, Elizabeth R, Carlos S, German H, Eduardo G. 2005 Incidence of and risk factors for Surgical-site Infections in a Peruvian Hospital. Infect Control Hosp Epidemiol;26(15):473-7.

Horan TC, Andrus M, Dudeck M A. 2008 CDC/NHSN surveillance definition of health care-associated infection and criteria for specific types of infections in the acute care setting. Am J Infect Control;36(15):309-32

Horan TC, Gaynes RP, Martone WJ, Jarvis WR, Emori TG. 1992 CDC definitions of nosocomial surgical site infections, 1992: a modification of CDC definitions of surgical wound infections. Infect Control Hosp Epidemiol;13(10):606-8.

Makoutodé M, Birintanya N, Djigbénoudé O, Paraïso NM, Ouendo E-M, Agueh V. 2002 Etude de l'incidence de l'infection des plaies post-opératoires au centre hospitalier départemental de l'Ouémé et du Plateau au Bénin. Le Bénin Médical;21:59-62.

National Nosocomial Infections Surveillance (NNIS) System.1996. National Nosocomial Infections Surveillance (NNIS) Report, Data Summary from October 1986-April 1996, Issued May 1996. Am J Infect Control;24(5):3808 
Oni A A, Ewete AF, Gbadja AT, Kolade AF, Mutiu WB, Adeyemo DA, et al. 2006 Nosocomial infections: surgical site infection in UCH Ibadan (Nigeria). Nigerian Journal of Surgical Research;8(1-2):19-23.

Ouédraogo A-S , Somé DA, Dakouré PWH , Sanon BG, Birba E, Poda GEA , Kambou T. 2011 Profil bactériologique des infections du site opératoire au centre hospitalier universitaire Souro Sanou de Bobo Dioulasso. Med Trop; 71 :49-52

Ruef C, Pittet D. 1998 Épidémiologie et prévention des infections du site chirurgical : progrès et problèmes. Méd et Hyg;56(2225):1846-52.

Satyanarayana V, Prashanth H V, Basavaraj B, Kavyashree A N. 2011 Study of surgical site infections in abdominal surgeries. Journal of Clinical and Diagnostic Research;29(17):935-9.

Umesh SK, Fereirra AMA, Kulkarni MS, Motghare DD. 2008. A prospective study of surgical site infections in a teaching hospital in Goa. Indian J Surg ;70(3):120-4.

Walter PW, Marcel Z, Stefan R, Chantal F-M, Heidi M, Rachel R, et al. 2008. Infect Control and Hosp Epidemiol;29(17):623-9.

Wassef MA, Hussein A, Abdul Rahman EM, El-Sherif RH. 2012. A prospective surveillance of surgical site infections: Study for efficacy of preoperative antibiotic prophylaxis. Afr J Microbiol Res;6(12):3072-8. 